\title{
ADAPTIVE TOPOLOGY OPTIMIZATION FOR INNOVATIVE 3D PRINTED METAMATERIALS
}

\author{
Daniele di Cristofaro $^{1}$, Chiara Galimberti ${ }^{1}$, Daniele Bianchi ${ }^{2,3}$, Raffaele Ferrante $^{*, 4,3}$, \\ Nicola Ferro ${ }^{5}$, Marco Mannisi ${ }^{3}$ and Simona Perotto ${ }^{5}$ \\ ${ }^{1}$ Politecnico di Milano \\ Piazza L. da Vinci, 32, I-20133, Milano \\ \{chiara1.galimberti, daniele.dicristofaro\}@mail.polimi.it \\ 2 Dipartimento di Ingegneria Civile e Architettura, Università degli Studi di Pavia \\ via Adolfo Ferrata, 5, I-27100, Pavia \\ daniele.bianchi@unipv.it \\ ${ }^{3}$ Medere S.r.l. \\ via Monasterace, 43, I-00118, Roma \\ \{daniele.bianchi, raffele.ferrante, marco.mannisi\}@ medere.it \\ ${ }^{4}$ Dipartimento di Ingegneria Civile ed Ingegneria Informatica, Università Tor Vergata \\ via Politecnico, 1, I-00133, Roma \\ raffaele.ferrante@uniroma2.it \\ ${ }^{5}$ MOX - Dipartimento di Matematica, Politecnico di Milano \\ Piazza L. da Vinci, 32, I-20133, Milano \\ \{nicola.ferro, simona.perotto\}@ polimi.it
}

Key words: Topology optimization, inverse homogenization, anisotropic adapted grid, metamaterials, $3 \mathrm{D}$ printing.

\begin{abstract}
An adaptive method for designing the infill pattern of 3D printed objects is proposed. In particular, new unit cells for metamaterials are designed in order to match prescribed mechanical specifications. To this aim, we resort to topology optimization at the microscale driven by an inverse homogenization to guarantee the desired properties at the macroscale. The whole procedure is additionally enriched with an anisotropic adaptive generation of the computational mesh. The proposed algorithm is first numerically verified both in a mono- and in a multi-objective context. Then, a mechanical validation and 3D manufacturing through fused-model-deposition are carried out to assess the feasibility of the proposed design workflow.
\end{abstract}

\section{MOTIVATIONS}

Recently, the developing of innovative manufacturing technologies has involved the industry in numerous fields (e.g., automotive, aerospace, medical industry) [4, 23]. Among the different innovations, the Additive Manufacturing (AM) has revolutionized the way to think the production [2]. In fact, the AM has allowed the production of objects with complex geometries in a simple way, overcoming the constraints 
imposed by traditional technologies, such as material removal, forming and tooling. Moreover, the AM, thanks to the versatility of the manufacturing system, has allowed to optimize the design on each specific case following the mechanical and structural requirements [31]. Generally, in the innovative manufacturing technologies, the structural optimization is driven by trial-and-error approaches. To minimize the waste of material and time, a more rigorous approach is represented by topology optimization methods that allow for increasingly efficient designs. The optimization procedures aim at identifying an optimal material distribution within a given design domain, according to prescribed requirements [5]. In this study, a design method for production through additive manufacturing is proposed involving topology optimization properly combined with inverse homogenization to satisfy structural requirements. In particular, different unit cells, suitable to be produced via AM, are designed addressing different monoand multi-objective structural problem requirements. The cells developed are then subject to numerical verification, validation, and manufacturing through $3 \mathrm{D}$ printing.

\section{A MICROSTRUCTURAL DESIGN TECHNIOUE,}

To provide the design of new microstructures, we resort to an inverse homogenization approach $[3,27]$. The idea is to periodically repeat in the macro design domain a unit cell, $Y$, which is properly optimized in order to match a required property at the macroscale (for instance, in a mechanical, thermal or magnetic setting). Cell $Y$ is designed by resorting to a topology optimization procedure performed at the microscale. Among the several approaches available in the literature for topology optimization [26, 28], we adopt the Solid Isotropic Material with Penalization (SIMP) method [5]. In this context, a suitable power of an auxiliary fup the constitutive law gov function, taking values mediate values in the in combined with standard thesholdin of $\rho$.
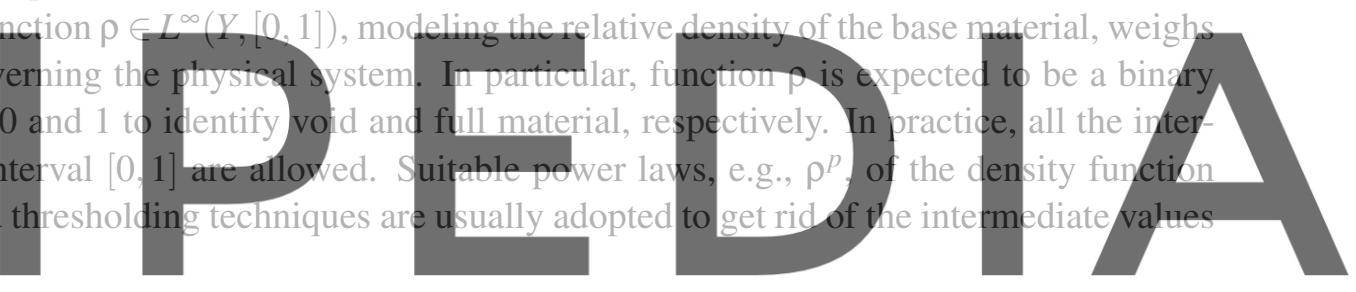

Thus, the formulation of a generic topology optimization problem solved in $Y$ reads

Register for free at https//www.scipedia.com to download the version without the watermark

$$
\min _{\rho \in L^{\infty}(Y,[0,1])} \mathcal{G}(\rho):\left\{\begin{array}{l}
a_{\rho}(u, w)=F_{\rho}(w) \forall w \in U \\
\int_{Y} \rho d Y \leq \alpha|Y| \\
\rho_{\min } \leq \rho \leq 1 .
\end{array}\right.
$$

In this formulation, $\mathcal{G}(\rho)$ is the goal quantity to be optimized; the first constraint is the weak form of the state equation, weighed by the density $\rho$; the first inequality imposes an upper bound on the maximum volume $|Y|$ of the cell, $\alpha$ being the allowed volume fraction; finally, the box constraint on $\rho$ guarantees the well-posedeness of the weak form by introducing a lower bound, $\rho_{\min }$, on the density. Problem (1) exhibits some well-known issues, such as the non uniqueness of the solution and the presence of intermediate densities (known as grayscale effect) [29]. Clearly, the definition of $\mathcal{G}(\rho), a_{\rho}$ and $F_{\rho}$ depends on the specific topology optimization problem, namely, on the application of interest. Next section is devoted to characterize the functional $\mathcal{G}(\rho)$ and the forms $a_{\rho}$ and $F_{\rho}$ when dealing with the design of microstructures. In particular, the microcell optimization is performed in a $2 \mathrm{D}$ setting and then converted into 3D unit cells via extrusion (for the details, see Section 4). 


\subsection{Direct and inverse homogenization approaches}

For both direct and inverse homogenization, we assume as a reference physical model the linear elasticity equation, characterized by the well-known stress-strain $(\sigma-\varepsilon)$ relation

$$
\sigma=\left[\begin{array}{l}
\sigma_{11} \\
\sigma_{22} \\
\sigma_{12}
\end{array}\right]=\left[\begin{array}{lll}
E_{1111} & E_{1122} & E_{1112} \\
E_{2211} & E_{2222} & E_{2212} \\
E_{1211} & E_{1222} & E_{1212}
\end{array}\right]\left[\begin{array}{c}
\varepsilon_{11} \\
\varepsilon_{22} \\
2 \varepsilon_{12}
\end{array}\right]=E \varepsilon,
$$

according to the Voigt notation in a 2D setting [14], with $E$ the stiffness tensor.

Direct homogenization is a standard technique used to incorporate the contribution at the microscale into the macroscale model by modifying the stiffness tensor. To this aim, it is standard to adopt the two-step procedure:

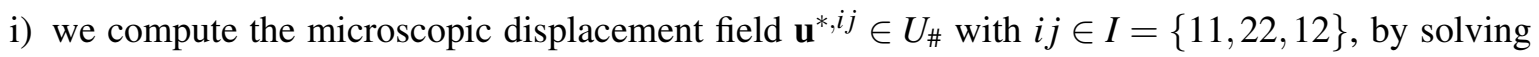
the elliptic equation

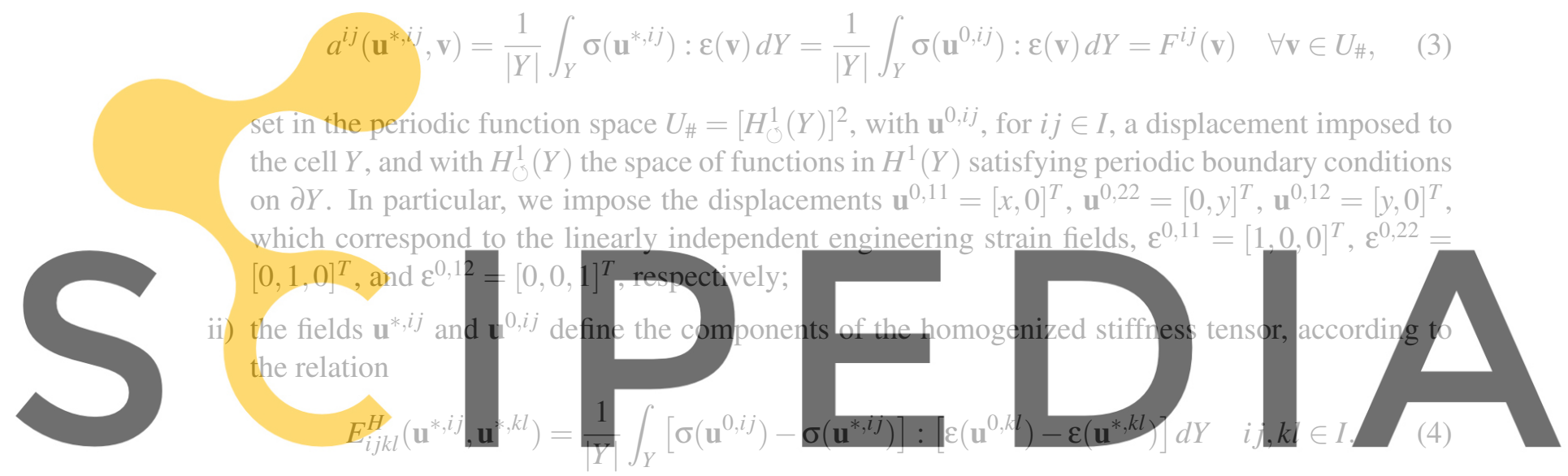

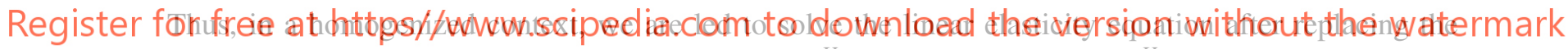
stress-strain relation in (2) with the new law $\sigma=E^{H} \varepsilon$, the stiffness tensor $E^{H}$ including now also the effect of the microscale.

Direct homogenization implies that $Y$ is known, whereas the effect of the repetition of the unit cell has to be determined at the macroscopic scale. Vice versa, the cell $Y$ represents the unknown of the inverse homogenization. According to [27], the optimal characterization of the unit cell is driven by topology optimization, so that the actual unknown is the distribution of material $\rho$ inside the design domain $Y$. Following the SIMP approach, the constitutive law $\sigma=E \varepsilon$ is changed into $\sigma_{\rho}=\rho^{p} E \varepsilon$, and relations (3) and (4) are replaced by

$$
\begin{gathered}
a_{\rho}^{i j}\left(\mathbf{u}^{*, i j}, \mathbf{v}\right)=\frac{1}{|Y|} \int_{Y} \rho^{p} \sigma\left(\mathbf{u}^{*, i j}\right): \varepsilon(\mathbf{v}) d Y=\frac{1}{|Y|} \int_{Y} \rho^{p} \sigma\left(\mathbf{u}^{0, i j}\right): \varepsilon(\mathbf{v}) d Y=F_{\rho}^{i j}(\mathbf{v}) \quad \forall \mathbf{v} \in U_{\#}, \\
E_{i j k l}^{H}\left(\mathbf{u}^{*, i j}, \mathbf{u}^{*, k l} ; \rho\right)=\frac{1}{|Y|} \int_{Y} \rho^{p}\left[\sigma\left(\mathbf{u}^{0, i j}\right)-\sigma\left(\mathbf{u}^{*, i j}\right)\right]:\left[\varepsilon\left(\mathbf{u}^{0, k l}\right)-\varepsilon\left(\mathbf{u}^{*, k l}\right)\right] d Y,
\end{gathered}
$$

with $i j, k l \in I$, respectively. At this level, the design variable $\rho$ is selected in $V_{\#}=H_{\circlearrowleft}^{1}(Y)$ to extend the periodic conditions on $\mathbf{u}^{*, i j}$ to the density function and to simplify the discussion below. 


\subsection{Topology optimization at the microscale}

The generic problem (1) is now particularized to the design of a unit cell $Y$. This implies to define $\mathcal{G}(\rho)$, $a_{\rho}$, and $F_{\rho}$ accordingly. Concerning the goal functional, we choose

$$
\mathcal{G}(\rho)=\mathcal{G}_{i j k l}\left(\mathbf{u}^{*, i j}, \mathbf{u}^{*, k l} ; \rho\right)=\frac{1}{2}\left[E_{i j k l}^{H}\left(\mathbf{u}^{*, i j}, \mathbf{u}^{*, k l} ; \rho\right)-E_{i j k l}^{G}\right]^{2},
$$

with $E_{i j k l}^{H}\left(\mathbf{u}^{*, i j}, \mathbf{u}^{*, k l} ; \rho\right)$ as in (6), and where $E_{i j k l}^{G}$ is the $i j k l$-th component of the user-defined goal stiffness tensor to be reached at the macroscale. The selected component allows us to control a specific physical quantity of interest as shown in the numerical verification. Moreover, different components of the stiffness tensor can be combined in the spirit of a multi-objective optimization.

As far as the weak form constraining problem (1), it exactly coincides with the three equations in (5).

In order to solve the minimization problem in (1), we have to compute the derivative of the goal functional with respect to the design variable $p$. For this purpose, we resort to an adjoint-based Lagrangian formulation, by introducing the augmented functional

$$
\mathcal{L}\left(\mathbf{u}^{*}, \lambda, \rho\right)=G_{i j k l}\left(\mathbf{u}^{*, i j}, \mathbf{u}^{*, k l} ; \rho\right)+\sum_{m n \in I}\left[a_{\rho}^{m n}\left(\mathbf{u}^{*, m n}, \lambda^{m n}\right)-F_{\rho}^{m n}\left(\lambda^{m n}\right)\right],
$$

for certain $i j, k l \in I$. The arguments $\mathbf{u}^{*}, \lambda$ of the augmented Lagrangian belong to the space $\left[U_{\#}\right]^{3}$, since collecting the three components $\mathbf{u}^{*}, m n, \lambda^{m n}$, with $m n \in I$, respectively, where $\lambda$ is the adjoint variable used

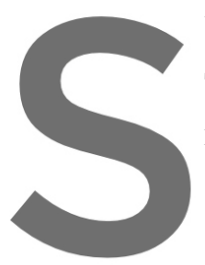
to impose the state equations.
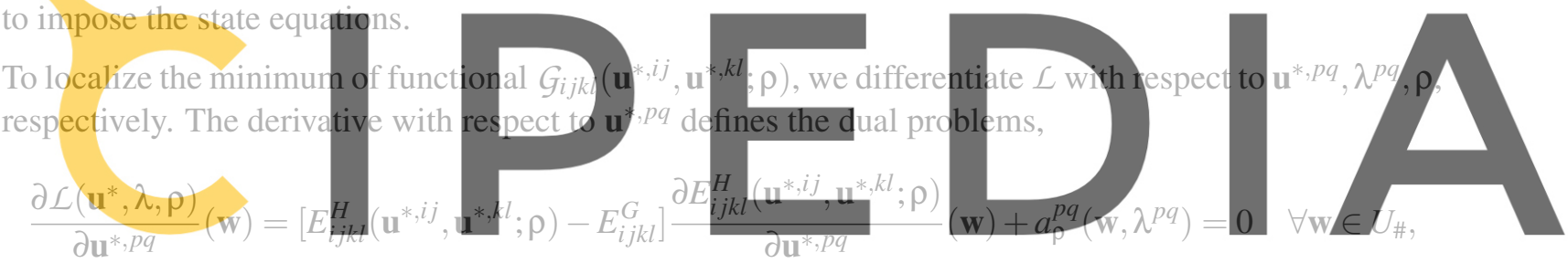

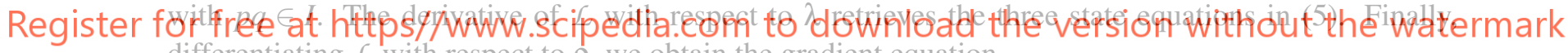
differentiating $\mathcal{L}$ with respect to $\rho$, we obtain the gradient equation.

$$
\begin{aligned}
\frac{\partial \mathcal{L}\left(\mathbf{u}^{*}, \lambda, \rho\right)}{\partial \rho}(\phi) & =\quad\left[E_{i j k l}^{H}\left(\mathbf{u}^{*, i j}, \mathbf{u}^{*, k l} ; \rho\right)-E_{i j k l}^{G}\right] \frac{\partial E_{i j k l}^{I I}\left(\mathbf{u}^{*, i j}, \mathbf{u}^{*, k l} ; \rho\right)}{\partial \rho}(\phi) \\
& +\sum_{m n \in I} \frac{1}{|Y|} \int_{Y} p \rho^{p-1}\left[\sigma\left(\mathbf{u}^{*, m n}\right)-\sigma\left(\mathbf{u}^{0, m n}\right)\right]: \varepsilon\left(\lambda^{m n}\right) \phi d Y \quad \forall \phi \in V_{\#} .
\end{aligned}
$$

The derivation of the dual and of the gradient equations can be generalized in a straightforward way when moving to a multi-objective context.

With a view to the numerical verification in the next section, the three state equations and dual problems, together with the gradient equation, have to be discretized. For this purpose, we introduce a conforming tessellation, $\mathcal{T}_{h}=\{K\}$, of the unit cell design domain consisting of triangular elements, and the associated space, $X_{h}^{1}(Y)$, of the affine finite elements. It is well-known that the choice of the computational mesh plays a crucial role in the topology optimization procedure. There is a strong dependence of the final layout on the selected grid in accordance with the non-uniqueness of the solution to problem (1). Moreover, other issues affect the design of the optimal structure, such as the formation of checkerboard 
patterns, related to the two-field (density-displacement) formulation, the staircase effect or the geometric complexity, due to the employment of a too coarse or too fine mesh [29]. Filtering techniques and ad hoc choices for the discrete spaces represent standard solution to these drawbacks [15]. More recently, a combination of SIMP with anisotropic mesh adaptation has been proposed as a possible remedy to the staircase effect and to the generation of complex geometries $[8,9,10,11,21,22]$. We adopt this approach, which, in addition, allows us to employ a linear discretization for both density and displacement, choosing $\rho_{h} \in V_{\#, h}$ and $\mathbf{u}_{h}^{*, i j} \in\left[V_{\#, h}\right]^{2}$, with $V_{\#, h}=V_{\#} \cap X_{h}^{1}(Y)$ and with $i j \in I$.

\section{VERIFICATION}

Following [10], with a view to an efficient numerical implementation of the methodology introduced in the previous section, we enrich the inverse homogenization procedure with an anisotropic adaptive management of the computational mesh.

\subsection{The theoretical background: an anisotropic error analysis}

To generate an adapted mesh following the material-void interface in the design of the unit cell, we resort to a metric-based approach, where each element $K$ of the computational mesh $\mathcal{T}_{h}$ is characterized by two lenghts, $\lambda_{i, K}$, and two orthonormal vectors, $\mathbf{r}_{i, K},(i=1,2)$ [12]. Such quantities constitute the metric $\mathcal{M}=\left\{\lambda_{i, K}, \mathbf{r}_{i, K}\right\}_{K \in \mathcal{T}_{h}}$, and are strictly related to the standard invertible affine map $T_{K}: \hat{K} \rightarrow K$ from the reference triangle $\hat{K}$ to the generic element $K$, changing the circle, $C$, circumscribing $\hat{K}$ into the ellipse, $\mathcal{E}$, circumscribing $K$. In particular, quantities $\lambda_{i, K}$ (with $\lambda_{1, K} \geq \lambda_{2, K}>0$ ) measure the lengths of the

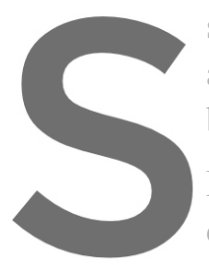
semiaxes of $\mathcal{E}$, while vect anisotropy of the element being identified by the 4 Metric $\mathcal{M}$ is predicted of the adapted mesh via
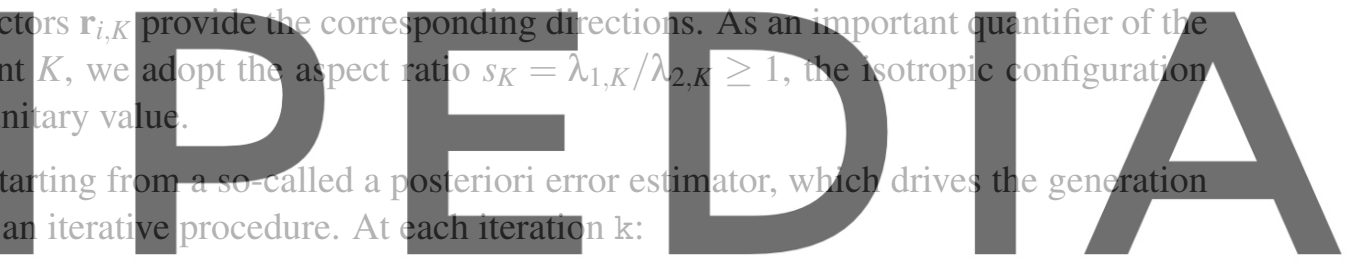

i) we compute the density $\rho_{h}^{\mathrm{k}}$, solution to the inverse homogenization problem in Section 2.2, on the Register for freesegt Thtstps//www.scipedia.com to download the version without the watermark

ii) we evaluate an a posteriori error estimator associated with $\rho_{h}^{\mathrm{k}}$;

iii) we construct the corresponding metric $\mathcal{M}^{\mathrm{k}}$;

iv) we generate the adapted mesh $\mathcal{T}_{h}^{\mathrm{k}+1}$ induced by the metric $\mathcal{M}^{\mathrm{k}}$.

We comment on ii)-iv), separately, and we drop the iteration index $\mathrm{k}$ to simplify the notation.

The a posteriori error estimator adopted at item ii) coincides with the anisotropic recovery-based estimator proposed in [19], namely

$$
\eta^{2}=\sum_{K \in \mathcal{T}_{h}} \eta_{K}^{2}, \quad \text { with } \quad \eta_{K}^{2}=\frac{1}{\lambda_{1, K} \lambda_{2, K}} \sum_{i=1}^{2} \lambda_{i, K}^{2}\left(\mathbf{r}_{i, K}^{T} G_{\Delta_{K}}\left(\mathbf{E}_{\nabla}\right) \mathbf{r}_{i, K}\right),
$$

where $\mathbf{E}_{\nabla}$ denotes the so-called recovered error, $\left[G_{K}(\mathbf{z})\right]_{s t}=\sum_{T \in \Delta_{K}} \int_{T} z_{s} z_{t}$ is a symmetric positive semidefinite matrix, with $s, t=1,2$ and $\left(z_{1}, z_{2}\right) \in\left[L^{2}(\Omega)\right]^{2}$, and $\Delta_{K}$ is the patch of elements associated with $K$. According to [33,34], $\mathbf{E}_{\nabla}$ coincides with the difference between the discrete gradient of the density, $\nabla \rho_{h}$, 
and a corresponding suitable reconstruction, $P\left(\nabla \rho_{h}\right)$. In the literature, several examples for the recovery operator $P$ are available (see, e.g., $[16,19,25,33,34]$ ), which consists of a projection or an average of the discrete gradient across a suitable patch of elements surrounding $K$. We adopt the area-weighed average over the patch $\Delta_{K}$,

$$
P\left(\nabla \rho_{h}\right)(\mathbf{x})=\left.\left|\Delta_{K}\right|^{-1} \sum_{T \in \Delta_{K}}|T| \nabla \rho_{h}\right|_{T}(\mathbf{x}) \quad \text { for any } \mathbf{x} \in K,
$$

$|\omega|$ denoting the measure of the generic domain $\omega \subset \mathbb{R}^{2}$. Estimator (8) has been already employed for several engineering applications with excellent results [6, 7, 20, 22, 24].

Estimator $\eta$ in (8) is successfully exploited to predict the metric $\mathcal{M}$ at item iii). The criteria driving the definition of $\mathcal{M}$ are the enforcement of a certain accuracy TOL on $\rho_{h}$, the minimization of the mesh cardinality, and the error equidistribution throughout the mesh. These requirements lead us to solve a constrained minimization problem on each element $K$, which is characterized by an explicit solution. In particular, first, the local estimator in (8) is scaled with respect to the area of $\Delta_{K}$, so that

$$
\eta_{K}^{2}=\lambda_{1, K} \lambda_{2, K}\left|\Delta_{\hat{K}}\right|\left[s_{K}\left(\mathbf{r}_{1, K}^{T} \hat{G}_{\Delta_{K}}\left(\mathbf{E}_{\nabla}\right) \mathbf{r}_{1, K}\right)+s_{K}^{-1}\left(\mathbf{r}_{2, K}^{T} \hat{G}_{\Delta_{K}}\left(\mathbf{E}_{\nabla}\right) \mathbf{r}_{2, K}\right)\right]
$$

where $\hat{G}_{\Delta_{K}}\left(\mathbf{E}_{\nabla}\right)$ is the scaled matrix $G_{\Delta_{K}}\left(\mathbf{E}_{\nabla}\right) /\left|\Delta_{K}\right|,\left|\Delta_{K}\right|=\lambda_{1, K} \lambda_{2, K}\left|\Delta_{\hat{K}}\right|$, and $\Delta_{\hat{K}}=T_{K}^{-1}\left(\Delta_{K}\right)$ is the pullback of the patch $\Delta_{K}$. Since, for the error equidistribution, $\eta_{K}^{2}$ is equal to a constant, minimizing the mesh cardinality turns out to be equivalent to maximize the area $\lambda_{1, K} \lambda_{2, K}\left|\Delta_{\hat{K}}\right|$ in (10), i.e., to minimize

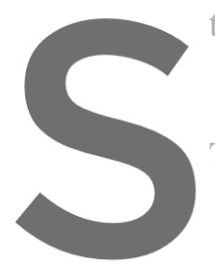
the quantity
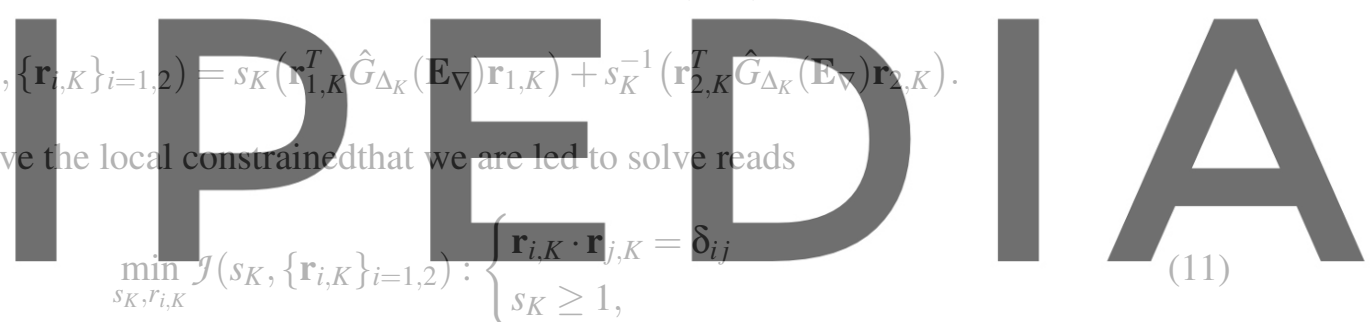

Register for free at https//www.scipedia.com to download the version without the watermark where $\delta_{i j}$ is the Kronecher symbol. Proposition 26 in [18] provides the explicit solution to problem (11), namely,

$$
s_{K}^{\mathrm{opt}}=\sqrt{\gamma_{1, K} / \gamma_{2, K}}, \quad \mathbf{r}_{1, K}^{\mathrm{opt}}=\mathbf{g}_{2, K}, \quad \mathbf{r}_{2, K}^{\mathrm{opt}}=\mathbf{g}_{1, K},
$$

with $\left\{\gamma_{i, K}, \mathbf{g}_{i, K}\right\}_{i=1,2}$ the eigen-pairs associated with matrix $\hat{G}_{\Delta_{K}}\left(\mathbf{E}_{\nabla}\right)$, with $\gamma_{1, K} \geq \gamma_{2, K}>0$ and $\left\{\mathbf{g}_{i, K}\right\}_{i=1,2}$ orthonormal vectors. Directions $\mathbf{r}_{i, K}^{\text {opt }}$ already represent two ingredients of the optical metric $\mathcal{M}$ that we are searching, whereas the length information, $\lambda_{i, K}^{\mathrm{opt}}$, is still gathered into the single quantity $s_{K}^{\mathrm{opt}}$. To derive lengths $\lambda_{1, K}^{\mathrm{opt}}, \lambda_{2, K}^{\mathrm{opt}}$ separately, we explicitly impose the error equidistribution, i.e.,

$$
\lambda_{1, K}^{\mathrm{opt}} \lambda_{2, K}^{\mathrm{opt}}\left|\Delta_{\hat{K}}\right|\left[s_{K}^{\mathrm{opt}}\left(\left(\mathbf{r}_{1, K}^{\mathrm{opt}}\right)^{T} \hat{G}_{\Delta_{K}}\left(\mathbf{E}_{\nabla}\right) \mathbf{r}_{1, K}^{\mathrm{opt}}\right)+\left(s_{K}^{\mathrm{opt}}\right)^{-1}\left(\left(\mathbf{r}_{2, K}^{\mathrm{opt}}\right)^{T} \hat{G}_{\Delta_{K}}\left(\mathbf{E}_{\nabla}\right) \mathbf{r}_{2, K}^{\mathrm{opt}}\right)\right]=\frac{\mathrm{TOL}^{2}}{\# \mathcal{T}_{h}},
$$

which yields

$$
\lambda_{1, K}^{\mathrm{opt}}=\gamma_{2, K}^{-1 / 2}\left(\frac{\mathrm{TOL}^{2}}{2 \# \mathcal{T}_{h}\left|\Delta_{\hat{K}}\right|}\right)^{1 / 2}, \quad \lambda_{2, K}^{\mathrm{opt}}=\gamma_{1, K}^{-1 / 2}\left(\frac{\mathrm{TOL}^{2}}{2 \# \mathcal{T}_{h}\left|\Delta_{\hat{K}}\right|}\right)^{1 / 2}
$$



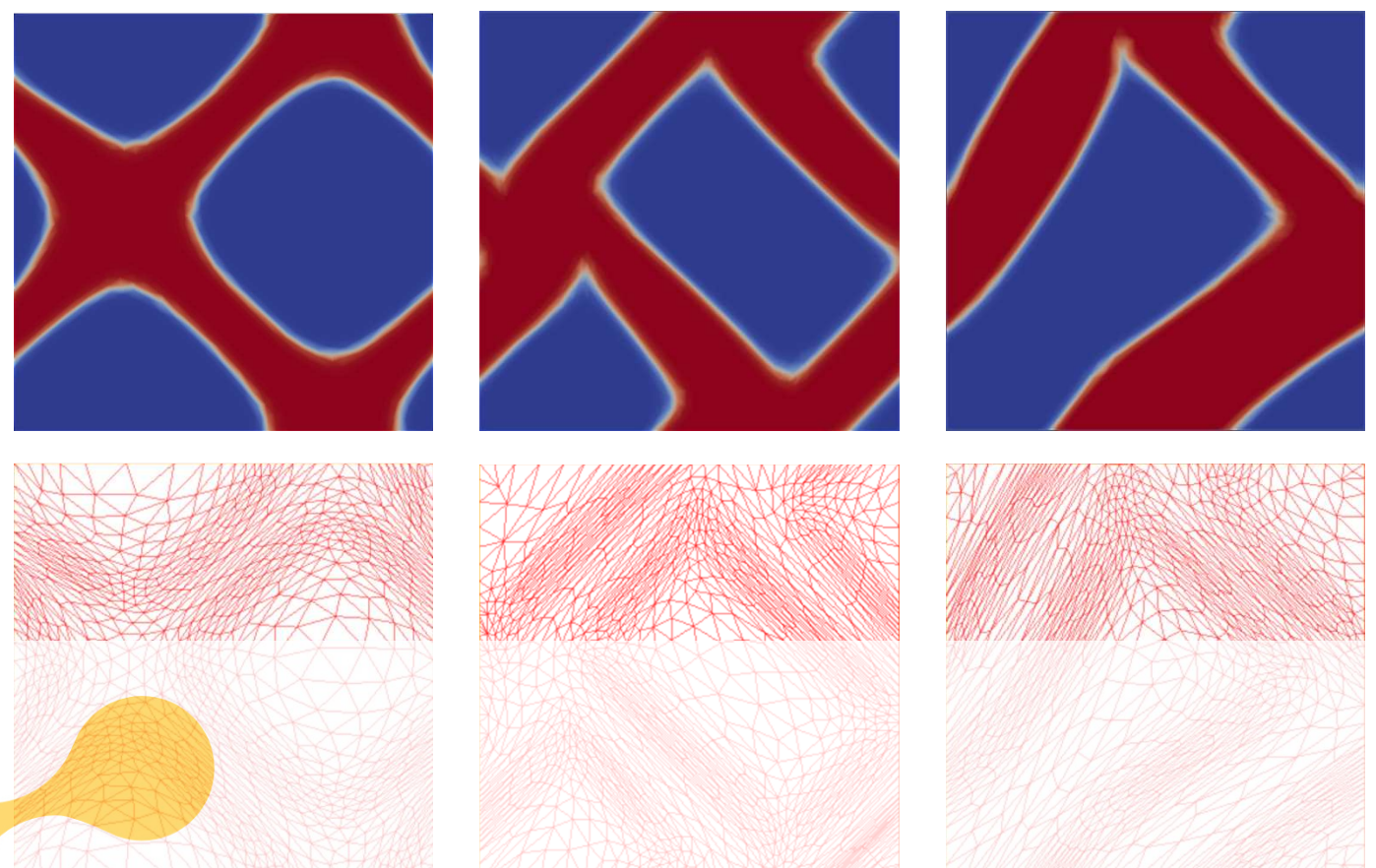

Figure 1: Optimized cells: density distribution (top) and associated anisotropic grid (bottom) for the mono- (left) and multi-objective $(\delta=0.5$ center; $\delta=0.8$ right) optimization.
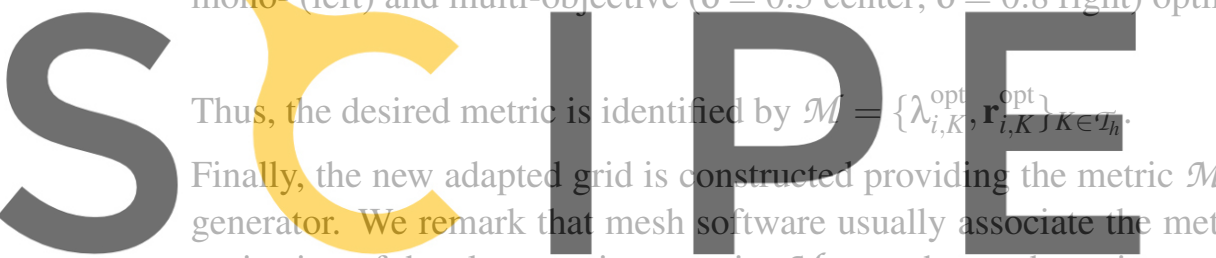

projection of the elementwise quantity $\mathcal{M}$ onto the mesh vertices could be demanded.

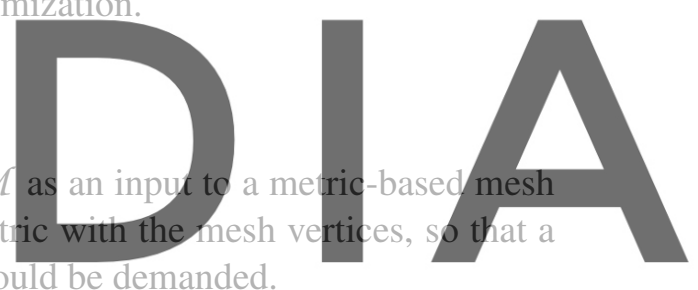

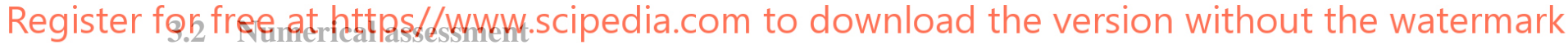

We apply the metric-based adaptive procedure in the previous section to the design of different unit cells.

In particular, we perform a mono- and a multi-objective optimization. To carry out the verification, we employ the microSIMPATY algorithm in [10], which alternates a topology optimization step with a mesh adaptation phase, until the stagnation of the mesh cardinality is reached or a maximum number, kmax, of (global) iterations is met. Among the input parameters expected by the procedure, we select the volume fraction $\alpha$, the tolerance, CTOL, to control the mesh stagnation, the tolerance, TOL, set by the user to drive the adaptive procedure, and the maximum number, OptMax, of iterations for the topology optimization. To perform the topology optimization, we resort to the Interior Point OPTimizer (IPOPT) package [32], while we adopt FreeFEM as solver, since particularly suited to manage an anisotropic adaptation of the mesh, being endowed with the metric-based mesh generator BAMG [13]. Finally, the power law used to penalize the intermediate densities according to the SIMP approach is set to 4 for all the numerical simulations below.

More technical details about the mechanical properties of the designed unit cells will be provided in the next section. 
Concerning the mono-objective cell design, we identify the goal functional $\mathcal{G}(\rho)$ with $\mathcal{G}_{1212}\left(\mathbf{u}^{*, 12}, \mathbf{u}^{*, 12} ; \rho\right)$ according to definition (7). This choice corresponds to optimize the topology of $Y$ with respect to the shear stress. As reference value $E_{1212}^{G}$, we choose $0.6[\mathrm{~Pa}]$. The input parameters to microSIMPATY are set to $\alpha=0.5, k \max =200$, CTOL $=1 \mathrm{e}-4$, TOL $=1 \mathrm{e}-5$, OptMax $=35$ for the first three iterations and OptMax $=10$ for the remaining ones, while a random initial guess for the density is selected and then discretized on a uniform structured mesh consisting of 1800 elements.

The design algorithm breaks after 41 iterations with the output layout in Figure 1 (top-left), whereas the corresponding adapted mesh, constituted by 1572 triangles, is shown in the bottom-left panel. The mesh elements are stretched to follow the void-material interface, with a maximum aspect ratio $s_{K}^{\max }=29.13$.

As far as the multi-objective design optimization is concerned, we control a combination of the shear stress and of the component $E_{1111}$ of the stiffness tensor. Thus, the goal functional $\mathcal{G}(\rho)$ becomes

$$
\delta \mathcal{G}_{1111}\left(\mathbf{u}^{*, 11}, \mathbf{u}^{*, 11} ; \rho\right)+(1-\delta) \mathcal{G}_{1212}\left(\mathbf{u}^{*, 12}, \mathbf{u}^{*, 12} ; \rho\right),
$$

with $0 \leq \delta \leq 1$ the parameter tuning the combination of the two quantities of interest. The reference values for the two selected components of the stiffness tensor are $E_{1111}^{G}=0.03[\mathrm{~Pa}]$, and $E_{1212}^{G}=0.2[\mathrm{~Pa}]$. MicroSIMPATY algorithm is run with the same input parameters as in the previous check, and choosing $\delta=0.5$. The procedure stops after 93 iterations by providing the unit cell in Figure 1 (top-center) and the corresponding final adapted mesh in Figure 1 (bottom-center), constisting of 1644 elements. It turns out that the control of the additional component $E_{1111}$ modifies the layout associated with the shear stress only. Moreover, the anisotropic features of the mesh are more evident as confirmed by the value of the

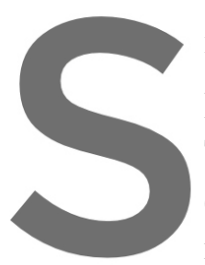
maximum aspect ratio Finally, we investigate To this aim, we choose convergence is reached a right). In contrast to the
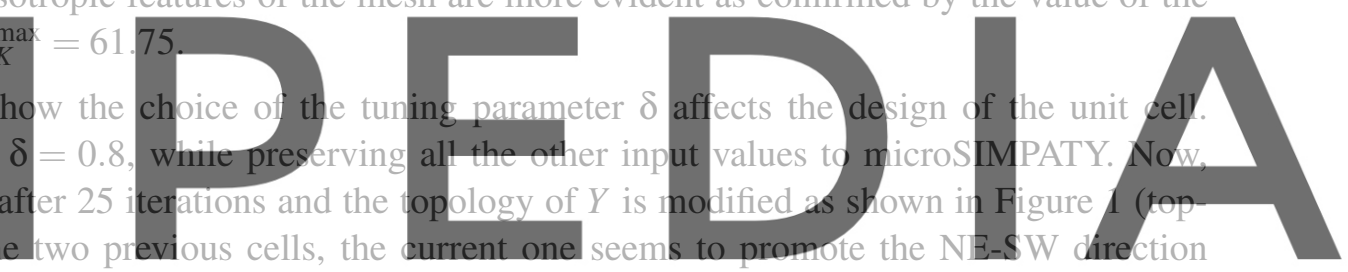

only. The associated grid in Figure 1 (bottom-right) is characterized by 1340 triangles with intermediate

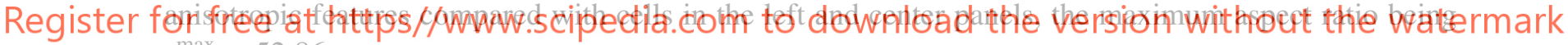
$s_{K}^{\max }=52.86$.

\section{VALIDATION}

In order to validate the results of the optimization process, the three cells in Figure 1 are compared in terms of mechanical behaviour. Moreover, to show the usability for AM, the optimized cells are manufactured via fused deposition modelling (FDM) through 3D printer.

\subsection{Mechanical validation}

Each 2D cell is regarded as a continuum square body with a side length $d$. The cell is composed of Thermoplastic Polyether-polyurethane Elastomer (TPE), a 3D printable filament chosen for the versatility of use. The mechanical behaviour of TPE material is assumed linearly elastic, homogeneous and isotropic. The equilibrium problem, related to optimized cells subject to load condition, is solved exploiting the finite-element capabilities of COMSOL Multiphysics [1]. Addressing a compression test to validate the mechanical behaviour of the optimized structures, the cells are constrained on the bottom boundary and a constant pressure $p$ is applied on the top boundary. A schematic representation for the mono-objective 


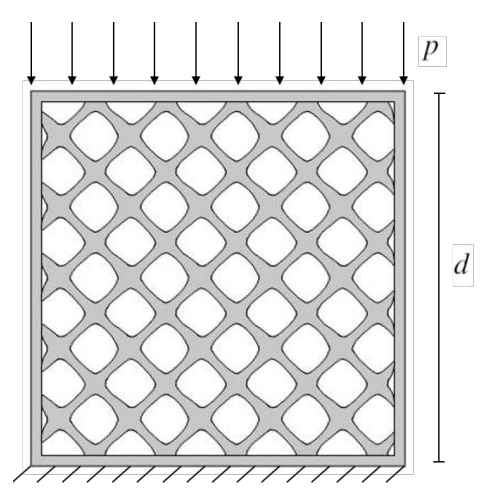

\begin{tabular}{lc}
\hline Data & \\
\hline Imposed pressure $p$ & $250 \mathrm{KPa}$ \\
Geometry side length $d$ & $5.5 \mathrm{~cm}$ \\
TPE Young's modulus & $45 \mathrm{MPa}$ \\
TPE Poisson's ratio & 0.49 \\
\hline
\end{tabular}

Figure 2: Generic graphical representation of the mono-objective optimization (left); specific data for the considered validation (right).

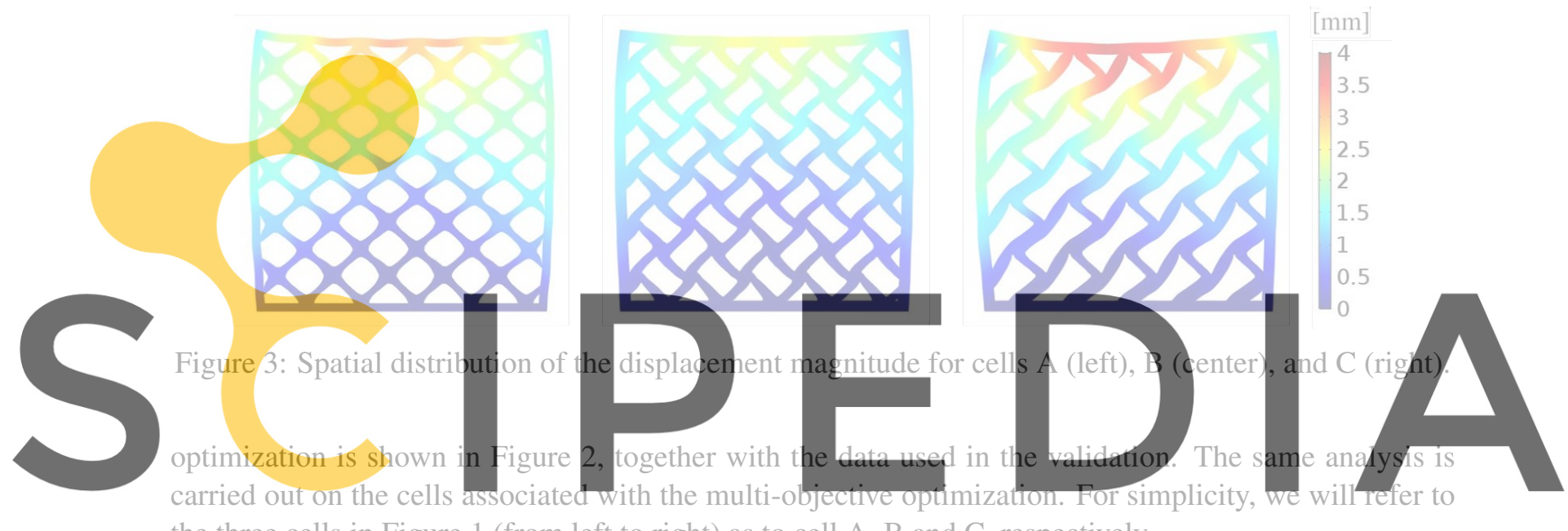
the three cells in Figure 1 (from left to right) as to cell A, B and C, respectively.

Register for free at https//www.scipedia.com to download the version without the watermark The performed experiment focuses on the mechanical response of the optimized cells. Figure 3 compares

the deformation of cells A, B, and C under the imposed load, in terms of spatial distribution of the displacement magnitude. It is evident that the same volume fraction leads to a very different mechanical behaviour when controlling diverse goal quantities. Cell B exhibits the lowest displacement magnitude, whereas cell $\mathrm{C}$ presents a not symmetric deformation. As far as the multi-objective optimization is concerned, we observe that cell $\mathrm{C}$ shows a $40 \%$ increment in the displacement magnitude peak with respect to cell B.

To characterize the loading-transfer mechanism within the structure, we investigate also the mechanical behaviour of the three cells in terms of the von Mises stress distribution under the imposed pressure (see Figure 4). The non-symmetric response of cell $\mathrm{C}$ is evident also for the von Mises stress, which reaches the highest values along the vertical boundary of the structure and in correspondence with the internal struts, when compared with cells A and B. 

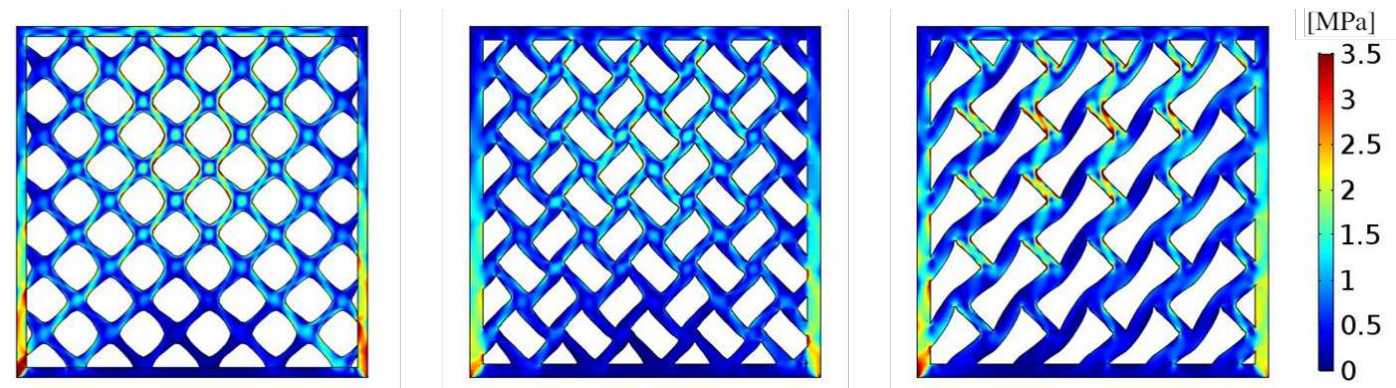

Figure 4: Spatial distribution of the von Mises stress for cells A (left), B (center), and C (right).

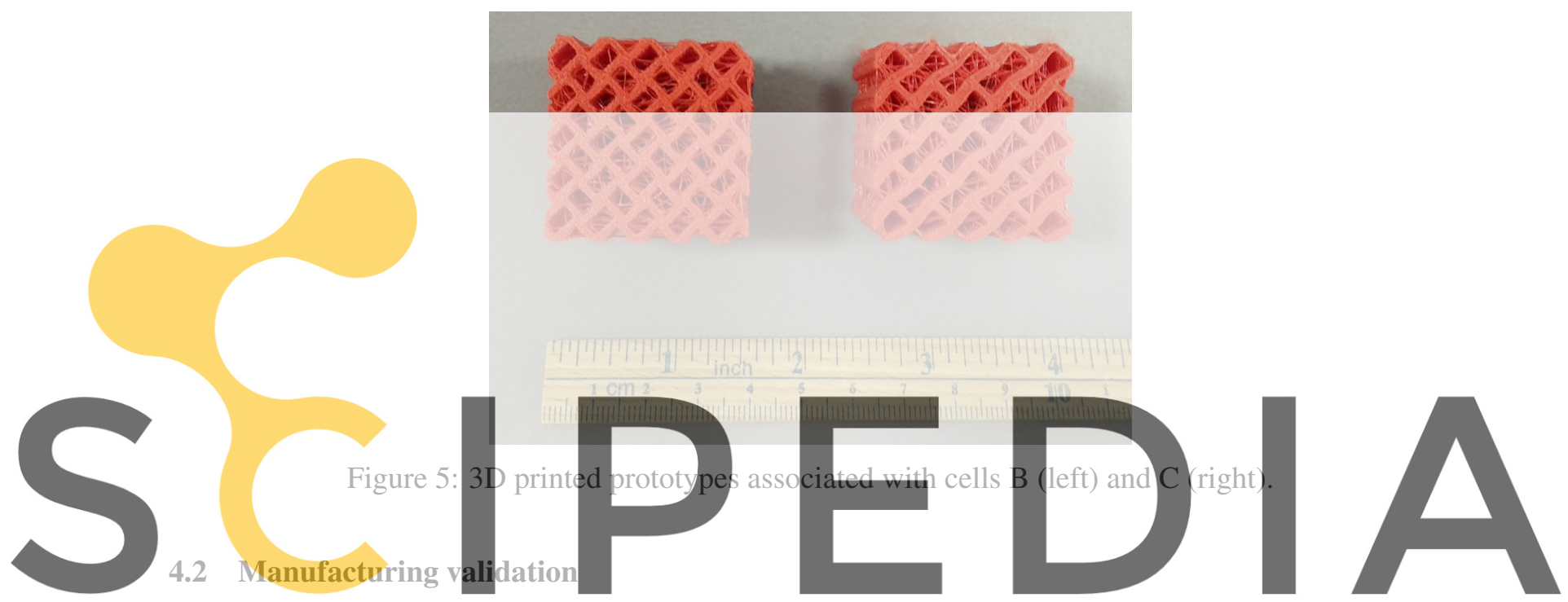

To validate the feasibility of the designed 2D cells, an axial extrusion was conducted to obtain a 3D

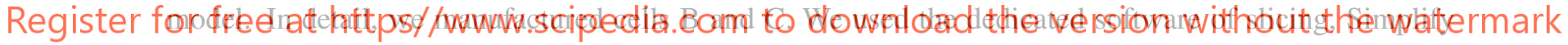

3D, to generate a piece of code for the 3D printer. Different slicing parameters and profiles were tested

to obtain the optimal result in terms of reliability and quality of production. The flexible TPE filament

Filaflex, with a shore A equal to 82 (Filaflex $82 \mathrm{~A}$ ), has been selected to manufacture the prototypes (see Figure 5). This material has optimal resistance to elongation and abrasion as well as high tensile strength. These properties make Filaflex $82 \mathrm{~A}$ a perfect material for the production of flexible and resistant parts. After several tests, the main printing parameters were set as following: print speed $2200 \mathrm{~mm} / \mathrm{min}$, extruder temperature $235^{\circ} \mathrm{C}$, cooling $60 \%$, no-retraction, top/bottom/outline perimeters equal to 3 . Each model took approximately 4 hours of printing. The produced 3D samples prove the manufacturability of the cells showing that AM technologies can fill the gap between the numerical optimization method and application.

\section{CONCLUSIONS}

In this work, a structural design technique for additive manufacturing technologies, combining anisotropic mesh adaptation, topology optimization and inverse homogenization, is proposed. The developed methodology has been tested to accomplish both mono- and multi-objective mechanical requirements. The op- 
timized cells have been computationally validated and experimentally verified. Moreover, the effective applicability of the proposed technique in AM has been checked by manufacturing corresponding prototypes via $3 \mathrm{D}$ printing.

Future works will address specific applications in the field of biomedical engineering where AM technologies can exploit the potential for patient-specific device production as just demonstrated in the prototyping of innovative custom insoles [17,30]. We also highlight that the proposed algorithm is completely general and can be successfully exploited in different applicative contexts.

\section{ACKNOWLEDGMENTS}

The financial support of INdAM-GNCS Projects 2020 is gratefully acknowledged by Simona Perotto. Nicola Ferro thanks Fondazione Fratelli Confalonieri for the awarded grant.

\section{References}

[1] COMSOL Multiphysics ${ }^{\circledR}$. Version 5.6, www.comsol.com, COMSOL AB, Stockholm, Sweden.

[2] B. Ahuja, M. Karg, and M. Schmidt. Additive manufacturing in production: challenges and opportunities. In Laser $3 d$ manufacturing II, volume 9353, page 935304. International Society for Optics and Photonics, 2015.

[3] E. Andreassen and C. S. Andreasen. How to determine composite material properties using numerical homogenization. Comput. Mater. Sci., 83:488-495, 2014.

[4] F. Auricchio and S. Marconi. 3d printing: clinical applications in orthopaedics and traumatology. EFORT open reviews, 1(5):121-127, 2016.

[5] M. P. Bendsøe and O. Sigmund. Topology Optimization - Theory, Methods and Applications. Springer-Verlag, Berlin, 2003.

[6] B. Esfandiar, G. Porta, S. Perotto, and A. Guadagnini. Anisotropic mesh and time step adaptivity for solute transport modeling in porous media. In S. Perotto and L. Formaggia, editors, New Challenges in Grid Generation and Adaptivity for Scientific Computing, volume 5 of SEMA SIMAI Springer Ser., pages 231-260. Springer, Cham, 2015.

[7] P. E. Farrell, S. Micheletti, and S. Perotto. An anisotropic Zienkiewicz-Zhu-type error estimator for 3D applications. Int. J. Numer. Meth. Engng, 85(6):671-692, 2011.

[8] N. Ferro, S. Micheletti, and S. Perotto. POD-assisted strategies for structural topology optimization. Comput. Math. Appl., 77(10):2804-2820, 2019.

[9] N. Ferro, S. Micheletti, and S. Perotto. Compliance-stress constrained mass minimization for topology optimization on anisotropic meshes. SN Applied Sciences, 2:1196, 2020.

[10] N. Ferro, S. Micheletti, and S. Perotto. Density-based inverse homogenization with anisotropically adapted elements. In A. Corsini, S. Perotto, G. Rozza, and H. van Brummelen, editors, Numerical Methods for Flows, volume 132 of Lect. Notes Comput. Sci. Eng., pages 211-221. Springer Cham, 2020.

[11] N. Ferro, S. Micheletti, and S. Perotto. An optimization algorithm for automatic structural design. Comput. Methods Appl. Mech. Engrg., 372:113335, 2020. 
[12] L. Formaggia and S. Perotto. New anisotropic a priori error estimates. Numer. Math., 89(4):641667, 2001.

[13] F. Hecht. New development in FreeFem++. J. Numer. Math., 20(3-4):251-265, 2012.

[14] P. Helnwein. Some remarks on the compressed matrix representation of symmetric second-order and fourth-order tensors. Comput. Methods Appl. Mech. Engrg., 190(22-23):2753-2770, 2001.

[15] B. S. Lazarov and O. Sigmund. Filters in topology optimization based on Helmholtz-type differential equations. Internat. J. Numer. Methods Engrg., 86(6):765-781, 2011.

[16] G. Maisano, S. Micheletti, S. Perotto, and C. L. Bottasso. On some new recovery-based a posteriori error estimators. Comput. Methods Appl. Mech. Engrg., 195(37-40):4794-4815, 2006.

[17] M. Mannisi, A. Dell'Isola, M. S. Andersen, and J. Woodburn. Effect of lateral wedged insoles on the knee internal contact forces in medial knee osteoarthritis. Gait \& Posture, 68:443-448, 2019.

[18] S. Micheletti and S. Perotto. Reliability and efficiency of an anisotropic Zienkiewicz-Zhu error estimator. Comput. Methods Appl. Mech. Engrg., 195(9-12):799-835, 2006.

[19] S. Micheletti and S. Perotto. Anisotropic adaptation via a Zienkiewicz-Zhu error estimator for 2D elliptic problems. In G. Kreiss, P. Lötstedt, A. Målqvist, and M. Neytcheva, editors, Numerical Mathematics and Advanced Applications, pages 645-653. Springer-Verlag Berlin Heidelberg, 2010.

[20] S. Micheletti, S. Perotto, and P. E. Farrell. A recovery-based error estimator for anisotropic mesh adaptation in CFD. Bol. Soc. Esp. Mat. Apl. SeMA, 50:115-137, 2010.

[21] S. Micheletti, S. Perotto, and L. Soli. Ottimizzazione topologica adattativa per la fabbricazione stratificata additiva, 2017. Italian patent application No. 102016000118131, filed on November 22, 2016 (extended as Adaptive topology optimization for additive layer manufacturing, International patent application PCT No. PCT/IB2017/057323).

[22] S. Micheletti, S. Perotto, and L. Soli. Topology optimization driven by anisotropic mesh adaptation: Towards a free-form design. Comput. \& Structures, 214:60 - 72, 2019.

[23] H. Mohrbacher, M. Spöttl, and J. Paegle. Innovative manufacturing technology enabling light weighting with steel in commercial vehicles. Adv. Manuf., 3(1):3-18, 2015.

[24] G. M. Porta, S. Perotto, and F. Ballio. Anisotropic mesh adaptation driven by a recovery-based error estimator for shallow water flow modeling. Internat. J. Numer. Methods Fluids, 70(3):269299, 2012.

[25] R. Rodríguez. Some remarks on Zienkiewicz-Zhu estimator. Numer. Methods Partial Differential Equations, 10(5):625-635, 1994.

[26] G. I. N. Rozvany. A critical review of established methods of structural topology optimization. Struct. Multidiscip. Optim., 37(3):217-237, 2009.

[27] O. Sigmund. Materials with prescribed constitutive parameters: an inverse homogenization problem. Internat. J. Solids Structures, 31(17):2313-2329, 1994.

[28] O. Sigmund and K. Maute. Topology optimization approaches. Struct. Multidiscip. Optim., 48(6):1031-1055, 2013. 
[29] O. Sigmund and J. Petersson. Numerical instabilities in topology optimization: a survey on procedures dealing with checkerboards, mesh-dependencies and local minima. Struct. Optim., 16(1):68$75,1998$.

[30] V. Srivastava and H. Gaur. Revolutionary development in orthopedic insole by additive manufacturing. J. Crit. Rev., 7(4):1943-1947, 2020.

[31] S. A. Tofail, E. P. Koumoulos, A. Bandyopadhyay, S. Bose, L. O’Donoghue, and C. Charitidis. Additive manufacturing: scientific and technological challenges, market uptake and opportunities. Mater. Today, 21(1):22-37, 2018.

[32] A. Wächter and L. T. Biegler. On the implementation of an interior-point filter line-search algorithm for large-scale nonlinear programming. Math. Program., 106(1, Ser. A):25-57, 2006.

[33] O. C. Zienkiewicz and J. Z. Zhu. A simple error estimator and adaptive procedure for practical engineering analysis. Int. J. Numer. Meth. Engng, 24:337-357, 1987.

[34] O. C. Zienkiewicz and J. Z. Zhu. The superconvergent patch recovery and a posteriori error estimates. I: The recovery technique. Int. J. Numer. Meth. Engng, 33:1331-1364, 1992. 